\title{
Calibration of a PEM Detector with Depth of Interaction Measurement
}

\author{
G.-C. Wang, Student Member, IEEE J.S. Huber, Member, IEEE W.W. Moses, Senior Member, IEEE, \\ W.-S. Choong, Member, IEEE, and J.S. Maltz, Member, IEEE
}

\begin{abstract}
We present an in situ calibration technique for the LBNL Positron Emission Mammography (PEM) detector module that is capable of measuring depth of interaction (DOI). The detector module consists of 64 LSO crystals coupled on one end to a single photomultiplier tube (PMT) and on the opposite end to a 64 pixel array of silicon photodiodes (PD). The PMT provides an accurate timing pulse, the PDs identify the crystal of interaction, the sum provides a total energy signal and the $\Gamma=P D /(P D+P M T)$ ratio determines the depth of interaction. We calibrate using the ${ }^{176} \mathrm{Lu}$ natural background radiation of the LSO crystals. We determine the relative gain $(K)$ of the PMT and PD by minimizing the asymmetry of the $\Gamma$ distribution. We determine the depth dependence from the width of the $\Gamma$ distribution with optimal $K$. The performance of calibrated detector modules is evaluated by averaging results from 12 modules. The energy resolution is a function of depth ranging from $24 \%$ fwhm at the PD end to $51 \%$ fwhm at the PMT end, and the DOI resolution ranges from $6 \mathrm{~mm}$ fwhm at the PD end to $11 \mathrm{~mm}$ fwhm at the PMT end.
\end{abstract}

Index Terms-Biomedical imaging, calibration, detectors, depth of interaction (DOI), positron emission tomography (PET).

\section{INTRODUCTION}

$\mathrm{I}$ the design of PET detectors, there is a tradeoff between sensitivity and spatial resolution. Increasing crystal length to achieve higher sensitivity leads to deterioration of spatial resolution due to the increased probability of detecting a gamma photon that has penetrated through more than one crystal. By measuring the depth of interaction within a crystal, it is theoretically possible to overcome the need to compromise either of these detector parameters. Depth of interaction can be measured using light sharing by placing a photon detector at both ends of a continuous scintillator crystal [1-3]. In the calibration of detectors that utilize light sharing, gain balancing between detectors is an essential step. The most obvious way of calibrating depth in a detector utilizing light sharing is to use a collimated gamma beam to excite the scintillator crystal at known depths. However, this is not feasible for a fully assembled multicrystal detector,

Manuscript received November 15, 2003. This work was supported in part by the Director, Office of Science, Office of Biological and Environmental Research, Medical Science Division of the U.S. Department of Energy under contract No. DE-AC03-76SF00098, and in part by National Institutes of Health Grant No. RO1-CA67911.

The authors are with the Lawrence Berkeley National Laboratory, Mailstop 55-121, 1 Cyclotron Road, Berkeley, CA 94720 USA (telephone: 510-495-2944, e-mail: gcwang@lbl.gov). because the inner crystals are shielded from the excitation beam by their neighbors. One solution is to precalibrate each crystal before assembling the crystal array. However, in situ calibration is the only practical solution for ongoing calibration of the installed detectors. Such calibration is necessary to accommodate the effects of drift in photomultiplier tube gains, temperature sensitivity, and electronic aging.

The LBNL PEM detector consists of $643 \mathrm{~mm} \times 3 \mathrm{~mm} \times 30$ $\mathrm{mm}$ LSO crystals coupled to a single photomultiplier tube and an $8 \times 8$ silicon photodiode array [4]. The photomultiplier tube provides accurate timing. A custom PET readout IC (PETRIC) [5] amplifies the photodiode pulse signals and identifies the crystal of interaction by winner-take-all circuitry that chooses the crystal with the highest signal. The total energy is the sum of the photodiode and photomultiplier tube signals. Fig. 1 shows an assembled LBNL PEM detector.

For the LBNL PEM detector, the depth of interaction is determined by the ratio between the photodiode signal and the sum of the photodiode and photomultiplier tube signals [1]. Crystal dependent relative gains need to be determined to convert the photodiode and photomultiplier tube signals to the same energy scale. The challenge of this in situ calibration is to balance the gains between the photodiode and the photomultiplier tube signals, and obtain the transformation between the signal ratio and physical depth for each crystal. This paper describes a novel algorithm based on measuring the LSO background to determine the relative gain and depth dependence.

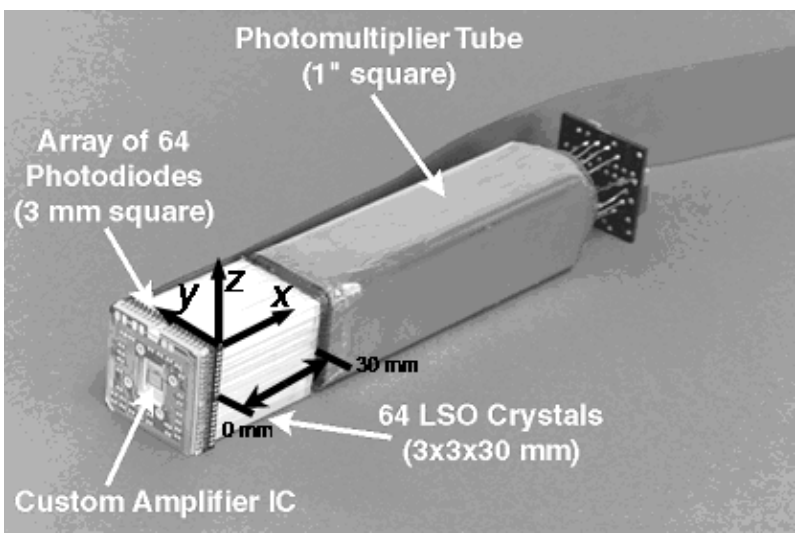

Fig. 1. Picture of the LBNL PEM module. The LSO crystals are sandwiched between a PMT and photodiode array. The $x$ here denotes the depth of interaction axis, which is defined to be zero at the photodiode end. 


\section{CAlibration Algorithm}

\section{A. Definitions}

Fig. 1 depicts the LBNL PEM module and our coordinate conventions. The LBNL PEM module has 64 LSO crystals, which need to be calibrated individually. For a given $i^{\text {th }}$ crystal, $P M T_{i}(x)$ and $P D_{i}^{\prime}(x)$ denote the uncorrected photopeak positions of the photomultiplier tube and the photodiode array signals when excited at a depth of $x$, respectively. The relative gain $K_{i}$ is defined as:

$$
K_{i}=\frac{P M T_{i}(x=30 \mathrm{~mm})}{P D_{i}^{\prime}(x=0 \mathrm{~mm})} .
$$

The gain-corrected photodiode bin is defined as:

$$
P D_{i}(x)=K_{i} \times P D_{i}^{\prime}(x) .
$$

The depth estimator $\Gamma_{\mathrm{i}}$ is defined as:

$$
\Gamma_{i}=\frac{P D_{i}(x)}{P M T_{i}(x)+P D_{i}(x)}=\frac{K_{i} \times P D_{i}^{\prime}(x)}{P M T_{i}(x)+K_{i} \times P D_{i}^{\prime}(x)},
$$

which is approximately linear in $x$ for all crystals [6].

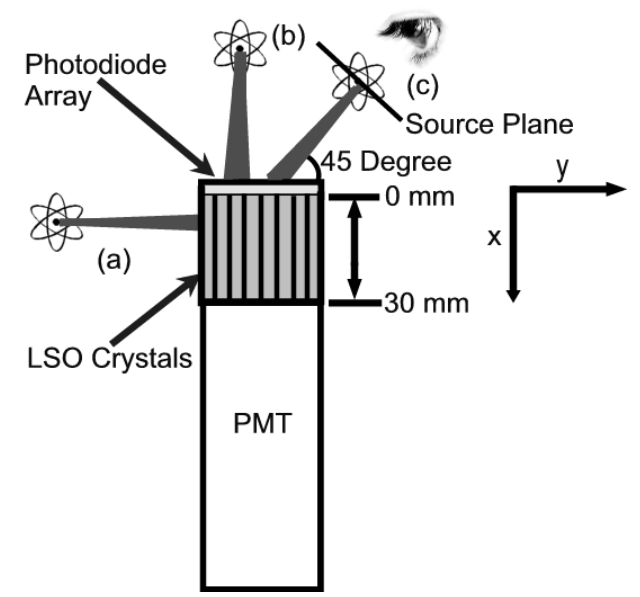

Fig. 2. Experimental setups used to validate and set up the calibration. An electronically collimated beam of $511 \mathrm{keV}$ gamma rays is incident (a) normal to the side, (b) normal to the face, and (c) at a 45-degree angle to the face of the LSO crystal array.

\section{B. Initial Setup}

\section{1) Photodiode Array Setup}

Each PETRIC chip processes signals from 64 photodiode pixels. The photocurrent from the photodiode array is collected and processed by the PETRIC to determine the address and signal amplitude of the interacting crystal. A fully functional PETRIC requires several internal registers and bias voltages to be configured correctly. Two bias voltages (controlled by digital potentiometers) adjust the rise and fall time of all the shapers in the PETRIC. In practice, they are manually adjusted to give a $1.2 \mu$ s peaking time which maximizes the signal to noise ratio of the pulse signal [5]. Preamplifier gains of the PETRIC are set to their maximum value for all channels. A reset current that compensates for photodiode leakage current is required for each channel to prevent the charge-sensitive-amplifier from saturating. For each channel, an electronic test pulse is injected and the reset current is increased until the output amplitude of the amplifier reaches a plateau. This procedure finds the reset current that minimizes the amplifier electronic noise. Channel pedestals (quiescent level of the amplifier output) are also measured and recorded.

2) Photomultiplier Tube Setup

A dedicated ASIC from CPS Innovations (Knoxville, TN) [7] processes the PMT pulse. "Singles" events are used to set up the PMT ASIC. A ${ }^{68} \mathrm{Ge}$ point source is positioned as shown in Fig. 2(b) to excite with $511 \mathrm{keV}$ gammas. The preamplifier gain of the PMT ASIC is adjusted until the photopeak of the pulse-height spectrum is close to one-third of the maximum ADC bin. This allows us to make full use of the ADC dynamic range. The constant-fraction-discriminator (CFD) threshold is set low enough so that the Compton shoulder in the pulse-height spectrum is observed, but high enough to avoid an excessive count rate.

\section{3) Readout Electronics Setup}

The electronic system described in [8] is used to read out the detectors. The system consists of 14 analog subsection boards, 4 detector head interface boards, and a coincidence controller. For calibration, the analog subsections are set to pass the interacting crystal address, photodiode and photomultiplier tube signal amplitudes to the coincidence controller. The coincidence controller is set to pass-through mode, in which it simply packs the data from the analog subsections into "singles" event words and sends these words to the host computer.

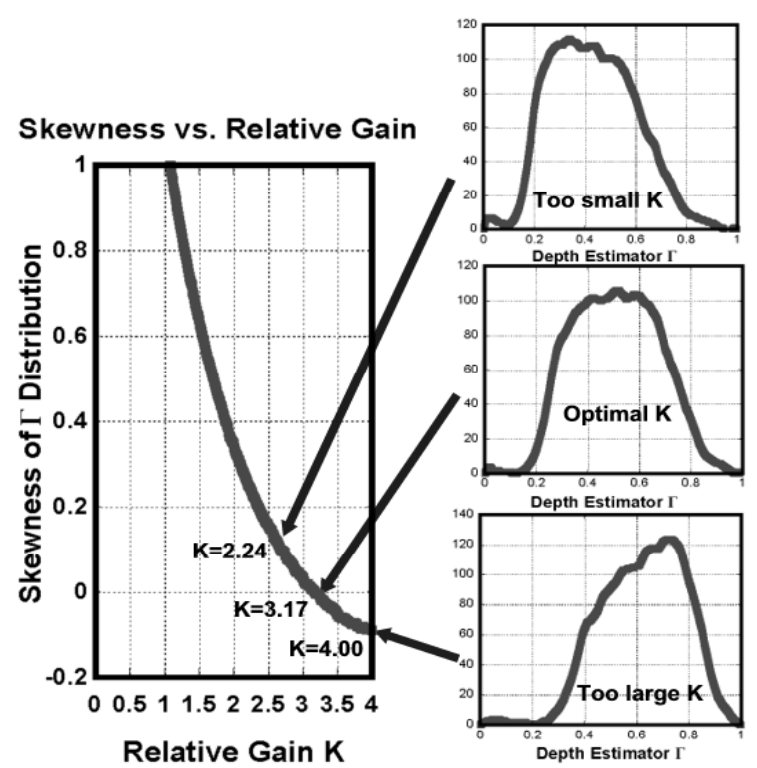

Fig. 3. Skewness as a function of relative gain $K$ for a typical crystal. The corresponding $\Gamma$ distributions are shown for specific $K$ values. The zero crossing of this crystal is at approximately $K=3.17$.

\section{Relative Gain}

LSO is naturally radioactive due to the presence of ${ }^{176} \mathrm{Lu}[9]$. The decay process consists of a beta decay followed by a gamma cascade. It is reasonable to assume that the ${ }^{176} \mathrm{Lu}$ is homogeneously distributed inside the LSO crystal. Based on this assumption, the radioactivity along the depth direction 
within the LSO crystal should be distributed symmetrically owing to the symmetric geometry of the crystal. (Escaping gammas at the edge of the crystal prevent the distribution from being completely uniform.)

"Singles" events are collected from the LSO background without an external source. The intensity of the natural background radiation is 300 decays $/ \mathrm{s} / \mathrm{cm}^{3}$. In our case, a 100 second acquisition time provides roughly 8000 events per crystal, which is enough for relative gain calibration. Since the depth estimator $\left(\Gamma_{i}\right)$ is approximately a linear function of position $x$, the histogram of $\Gamma_{i}$ distribution from the "singles" events of LSO background (with the correct relative gain $K_{i}$ ) should also be symmetric. Skewness (defined as the third central moment divided by the third power of the standard deviation of a distribution) describes the degree of asymmetry of a distribution, and a symmetric distribution exhibits zero skewness. With the optimal relative gain $K_{i}$, the skewness of the $\Gamma_{i}$ distribution falls to zero. This numerical one-dimensional zero crossing problem is solved by Newton's method. Fig. 3 shows an example of determining the optimal $K_{i}$. A too small $K_{i}$ leads to a left-skewed $\Gamma_{i}$ distribution, and a too large $K_{i}$ leads to the opposite. Only the proper $K_{i}$ corresponds to a symmetric $\Gamma_{i}$ distribution.

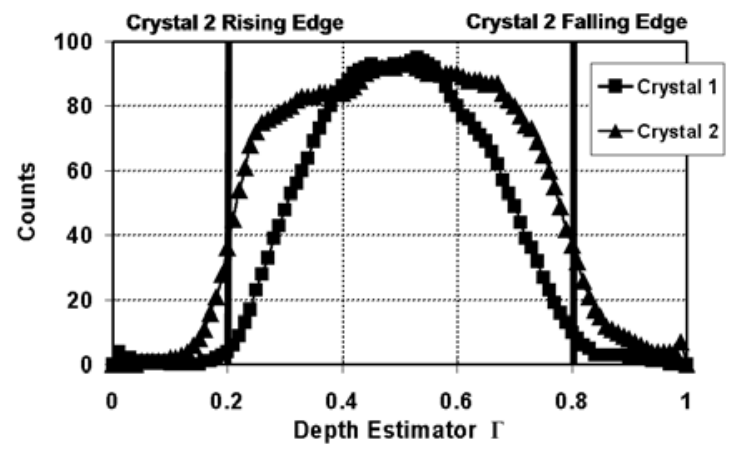

Fig. 4. Distributions of the depth estimator $\Gamma$ for two crystals using LSO background "singles". Crystals with larger depth dependence have wider $\Gamma$ distributions. The bars denote the rising and falling edges of crystal 2. A value of $\Gamma=0.3$ converts to the $0^{\text {th }}$ depth bin for crystal 1 but $1^{\text {st }}$ depth bin for crystal 2 .

\section{Depth Dependence}

After determining the optimal relative gain $K_{i}$, a linear transformation is needed to convert a given $\Gamma_{i}$ to the corresponding physical depth for the $i^{\text {th }}$ crystal. To attain this linear relation, we process the same raw set of LSO background data with the optimal $K_{i}$ and look at the $\Gamma_{i}$ distribution of background events. The physical edges of the crystal in the depth direction are highly correlated to the rising and falling edges of the background $\Gamma_{i}$ distribution (see Fig. 4). The rising and falling edges are identified empirically as the positions where the height of $\Gamma_{i}$ is one-third of the maximum count of the background $\Gamma_{i}$ distribution.

The goal of the LBNL PEM module is to resolve eight depth bins [8]. In other words, the goal is to divide the crystal into eight segments in the depth direction. This segmentation is equivalent to dividing the region between the rising and falling edge of the background $\Gamma_{i}$ distribution into eight segments. A given $\Gamma_{i}$ which is within the range of a segment is assigned to the corresponding depth bin. Mathematically, the depth bin for $i^{\text {th }}$ crystal is determined as follows:

$$
\operatorname{bin}_{i}=\text { Floor }\left(\frac{8 \times\left(\Gamma_{i}-\left(\Gamma_{R}\right)_{i}\right)}{\left(\Gamma_{F}\right)_{i}-\left(\Gamma_{R}\right)_{i}}\right),
$$

where $\left(\Gamma_{R}\right)_{i}$ and $\left(\Gamma_{F}\right)_{i}$ denote the rising and falling edges (both at $1 / 3$ maximum) of the background $\Gamma_{i}$ distribution, respectively. A typical $2 \mathrm{GHz} \mathrm{PC}$ can both collect the necessary data and compute the $K_{i}$ and depth factors for 64 crystals within 10 minutes.

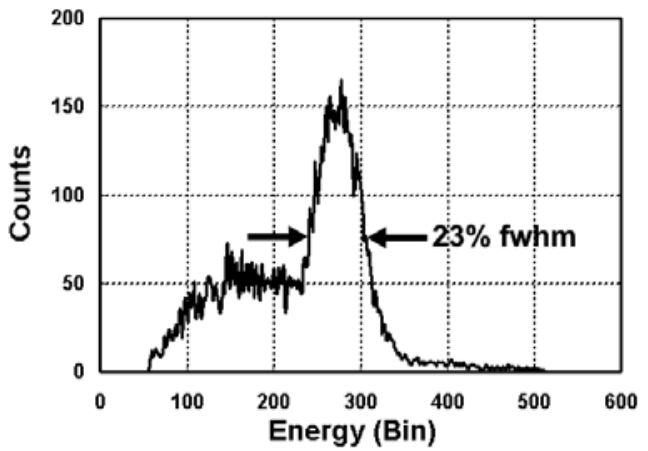

Fig. 5. Pulse-height spectrum of a typical crystal at $0^{\text {th }}$ depth bin (from 0 to $3.75 \mathrm{~mm}$ from the PD end). An energy resolution of $23 \% \mathrm{fwhm}$ is measured.

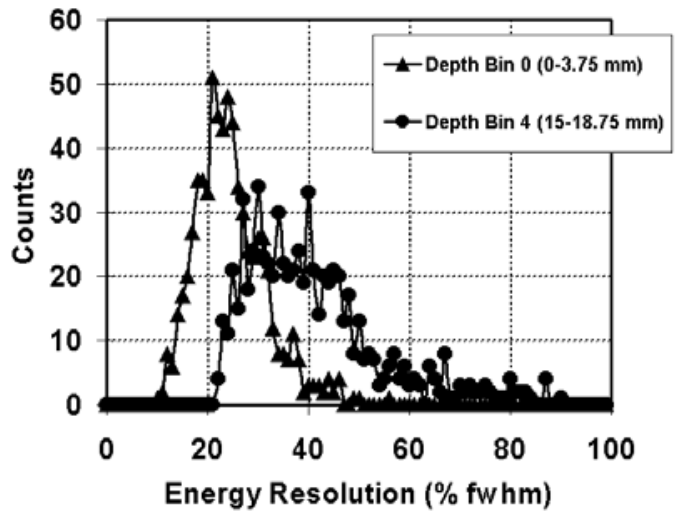

Fig. 6. Histograms of energy resolution (for two depths bins) for 692 crystals in 12 detector modules. The average energy resolution is $24 \%$ fwhm for the $0^{\text {th }}$ depth bin and $41 \%$ fwhm for the $4^{\text {th }}$ depth bin.

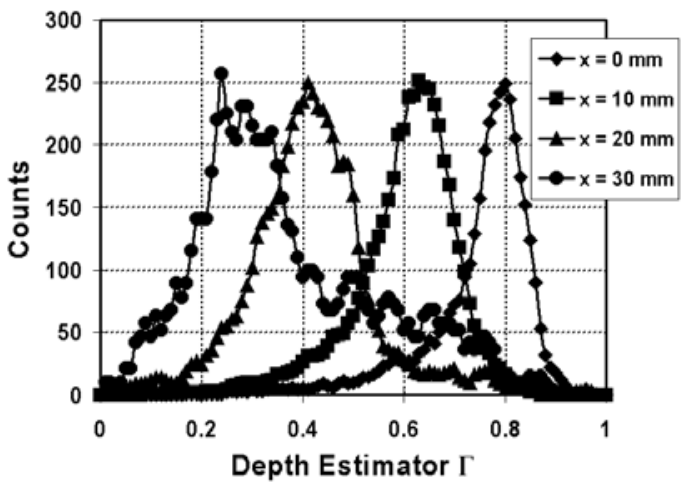

Fig. 7. $\Gamma$ distributions at four excitation depths for a single crystal. The fwhm of the $\Gamma$ distribution ranges from 0.11 to 0.27 bin, which corresponds to a 5.5 to $9.5 \mathrm{~mm}$ fwhm DOI resolution. 


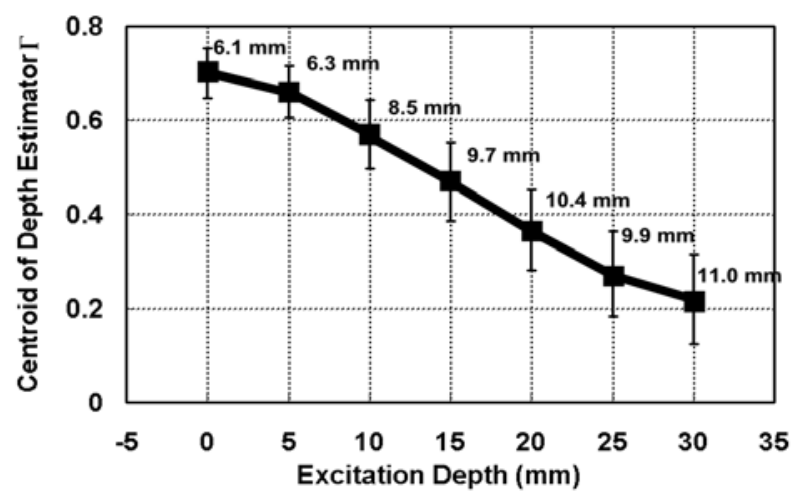

Fig. 8. Average centroid of $\Gamma$ and DOI are shown for 7 excitation depths. Measurements are averaged over 62 crystals. Selected events have $250<\mathrm{E}<750$ $\mathrm{keV}$ energy deposit. A $3 \mathrm{~mm}$ fwhm portion of the LSO crystal is excited.

\section{RESULTS AND VALIDATION}

\section{A. Energy Resolution}

"Singles" events from 692 crystals are taken with front excitation of a ${ }^{68} \mathrm{Ge}$ point source as illustrated in Fig. 2(b). Data are collected with the LBNL PEM electronic system and processed offline with the calibration parameters obtained from the algorithm described in Section II.C. Histogramming the sum of the PMT and gain-corrected PD signals produces pulse-height spectra. Fig. 5 shows the pulse-height spectrum of a typical crystal with interactions that occur in the $0^{\text {th }}$ depth bin (depth ranges from 0 to $3.75 \mathrm{~mm}$ from the $\mathrm{PD}$ end); the energy resolution is $23 \%$ fwhm.

The energy resolution is a function of depth. Fig. 6 shows the distribution of energy resolutions of 692 crystals for the $0^{\text {th }}$ and $4^{\text {th }}$ depth bins. The energy resolution degrades at higher depth bins because the signal to noise ratio of the photodiode is worse for events that occur near the PMT end of the crystal [6] (the photodiode noise is a constant but the signal depends on depth, so the signal to noise ratio is a function of depth). The average energy resolution ranges from $24 \%$ fwhm at the photodiode end $\left(0^{\text {th }}\right.$ depth bin) to $51 \%$ fwhm at the photomultiplier tube end ( $7^{\text {th }}$ depth bin). It is worth noting that an improper relative gain $K_{i}$ eliminates the photopeak. Therefore, a clear photopeak confirms that the relative gain $K_{i}$ is chosen properly.

\section{B. DOI Resolution}

We validate our calibration technique by exciting the modules from the side as illustrated in Fig. 2(a). An electronically collimated beam of $511 \mathrm{keV}$ gamma rays excites a $3 \mathrm{~mm}$ fwhm portion of the crystals. The collimated beam is scanned through the crystals in the $x$ direction using an XYZ stage in $5 \mathrm{~mm}$ increments from 0 to $30 \mathrm{~mm}$. Data from 62 crystals are taken to verify the excitation depth versus $\Gamma$ relation. The $511 \mathrm{keV}$ gamma beam is attenuated through the LSO crystals. Consequently, only the first three layers of the edge crystals record sufficient events to validate their calibration. Fig. 7 shows the $\Gamma_{i}$ distributions for a typical crystal with the module excited at four different depths $(0,10$, 20 , and $30 \mathrm{~mm}$ respectively). The linear movement (with $x$ ) of the centroid of the $\Gamma_{i}$ distribution is clearly shown. When averaging 62 crystals, we observe a highly linear relationship between the excitation depth and $\Gamma_{i}$, with a correlation coefficient of 0.99 (see Fig. 8).

The DOI resolution is defined as the fwhm of the $\Gamma_{i}$ distribution divided by the slope of the least square fit of $\Gamma_{i}$ versus excitation depth $x$ [6]. Because the width of the $\Gamma_{i}$ distribution depends on depth, the DOI resolution is a function of depth. The crystal shown in Fig. 7 has a DOI resolution of 5.5 to $9.5 \mathrm{~mm}$ fwhm. The average DOI resolution is $6.1 \mathrm{~mm}$ fwhm at the PD end and degrades to $11 \mathrm{~mm}$ fwhm at the PMT end (see Fig. 8). This degradation is also due to the decreased signal to noise ratio as described for energy resolution in the previous section. The DOI resolution has improved in comparison to a previous prototype module, which had 6 to 15 $\mathrm{mm}$ DOI resolution [4]. This improvement may be attributed to the lower electronic noise of the PETRIC.

\section{Module Performance}

To produce images that illustrate the performance of the calibrated DOI detector, data are taken with the setup as shown in Fig. 2(c). An electronically collimated beam of 511 $\mathrm{keV}$ gamma rays is incident at a 45-degree angle onto the face of the LSO crystal array. With the correct calibration, the position of the interaction pixels should lie on a 45-degree beam line in the $x-y$ plane. We process the data using the calibration factors derived from our algorithms, and determine the depth $\operatorname{bin}_{i}$ using equation (4). The events are assigned to three dimensional positions within the module, and the number of events at each position is stored in an $8 \times 8 \times 8$ array ( $8 \times 8$ crystals, and another 8 depth bins for each crystal). Only the events within a 250 to $750 \mathrm{keV}$ energy window are selected. Fig. 9 shows the $x-y$ projection image of this array. The 45-degree angle of the beam is clearly visible and the exponential attenuation of the beam with depth is also evident. The results validate the correctness of the assigned depth bins.

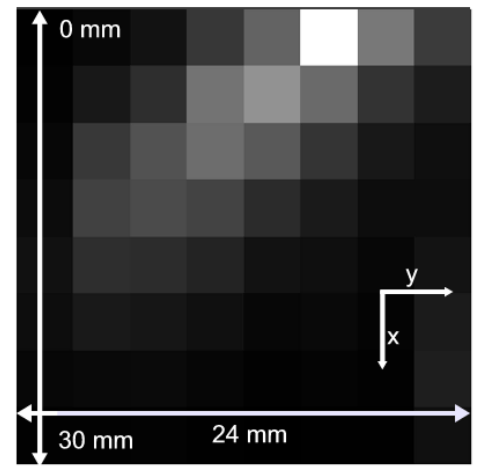

Fig. 9. Image of a collimated beam of gamma rays incident at 45 degrees onto the face of one detector module. The image shows the projection of the LSO array onto $x$ - $y$ plane. Selected events have $250<\mathrm{E}<750 \mathrm{keV}$ energy deposit.

Using the data that is displayed in Fig. 9, we can image the ${ }^{68} \mathrm{Ge}$ point source by backprojecting the array to the source plane as indicated in Fig. 2(c). Fig. 10(a) shows the image by backprojecting the whole $8 \times 8 \times 8$ array when DOI information 
is used. Fig. 10(b) shows the image reconstructed without DOI information by assigning all interactions to the $0^{\text {th }}$ depth bin, i.e. the front face of the LSO array. The reduced blurring due to the DOI capability is clearly visible.
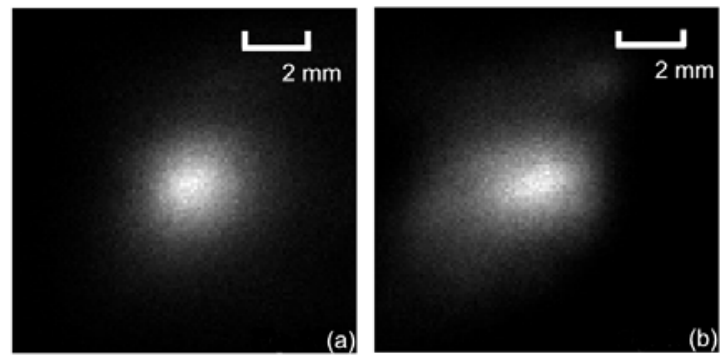

Fig. 10. Images of the ${ }^{68} \mathrm{Ge}$ point source. (a) The backprojected image with DOI information. The symmetry of the point source is preserved because of the DOI capability. (b) The backprojected image without DOI information. The image suffers from penetration artifacts. Both images are generated from the same set of data. The field of view is $1 \mathrm{~cm}$ square.

\section{DISCUSSION}

The calibration of a volumetric radiation detector poses unique challenges. Ideally, one would calibrate such a detector by exciting a single point inside the crystal array. However, the feasible option is to employ a collimated gamma beam to produce a line of excitation. There are two major problems with the collimated beam line: (1) the distribution of excitation is far from a perfect line because of scattering and attenuation; and (2) the geometry of the detector and camera usually limits the directions and positions of this beam. However, not all detector designs suffer this difficulty. For example, phoswich detectors that use layer dependent decay time to encode depth do not exhibit this problem.

Many prototype DOI module designs have been calibrated using side excitation [10, 11]. For these studies, depth calibration is straightforward since only a few crystals or channels are present. However, once the prototype is scaled up to a full-sized module and placed in a camera, an in situ calibration is required. The known activity distribution of the internal radioactive source (e.g., the ${ }^{176} \mathrm{Lu}$ in the LSO crystal) can be used to adjust and optimize the calibration parameters in volumetric detectors, which is especially helpful in designs that use the analog ratio of signals to measure depth.

When developing new scintillators, one could even add radioisotopes to non-radioactive scintillators to employ this calibration scheme. If so, it may be preferable to add radioisotopes that produce radiations that do not overlap with the energy window of the radiotracer being imaged.

\section{CONCLUSION}

We have developed and validated an in situ calibration algorithm for the LBNL PEM camera. This algorithm has three advantages: (1) no external source is needed for depth calibration; (2) data for calibration can be taken simultaneously for all crystals because the ${ }^{176} \mathrm{Lu}$ background radioactivity resides in every LSO crystal; and (3) little computation power is needed to process the data (a whole camera calibration can be done in several hours by a typical 2 $\mathrm{GHz} \mathrm{PC}$ ).

The performance of the calibrated modules has been evaluated. The results show that the average energy resolution ranges from $24 \%$ fwhm at the PD end to $51 \%$ fwhm at the PMT end. The DOI resolution is also a function of depth and ranges from $6 \mathrm{~mm}$ fwhm at the PD end to $11 \mathrm{~mm}$ fwhm at the PMT end. Since most of the gammas coming from a patient will interact with the crystal in the first three depth bins (where the modules have the best performance), the degradation of those resolutions will be less of an issue. Our calibration method can be applied to most PET detector designs that use analog ratio of signals to measure depth.

\section{ACKNOWLEDGMENT}

We thank CPS Innovations (Knoxville, TN) for providing electronics and technical assistance. This work was supported in part by the Director, Office of Science, Office of Biological and Environmental Research, Medical Science Division of the U.S. Department of Energy under contract No. DE-AC03-76SF00098, and in part by the National Institutes of Health, National Cancer Institute under Grant No. RO1-CA67911. Reference to a company or product name does not imply approval or recommendation by the University of California or the U.S. Department of Energy to the exclusion of others that may be suitable.

\section{REFERENCES}

[1] W. W. Moses and S. E. Derenzo, "Design studies for a PET detector module using a PIN photodiode to measure depth of interaction," IEEE Trans. Nucl. Sci., vol. 41, pp. 1441-1445, 1994.

[2] Y.P. Shao and S.R. Cherry, "A study of depth of interaction measurement using bent optical fibers," IEEE Trans. Nucl. Sci., vol. 46, pp. 618-623, 1999.

[3] Y. Shao, et al., "Design studies of a high resolution PET detector using APD arrays," IEEE Trans. Nucl. Sci., vol. 47, pp. 1051-1057, 2000.

[4] J. S. Huber, W. W. Moses, M. S. Andreaco, et al., "A LSO scintillator array for a PET detector module with depth of interaction measurement," IEEE Trans. Nucl. Sci., vol. 48, pp. 684-688, 2001.

[5] M. Pedrali-Noy, G. J. Gruber, B. Krieger, E. Mandelli, G. Meddeler, V. Rosso, W. W. Moses, "PETRIC - a positron emission tomography readout IC," IEEE Trans. Nucl. Sci., vol. 48, pp. 479-484, 2001.

[6] W. W. Moses, S. E. Derenzo and C. L. Melcher, "A room temperature LSO/PIN photodiode PET detector module that measures depth of interaction," IEEE Trans. Nucl. Sci., vol. 42, pp. 1085-1089, 1995.

[7] D. M. Binkley, M. J. Paulus, M. E. Casey, et al."A custom CMOS integrated circuit for PET tomograph front-end applications," Proceedings of The IEEE 1993 Nuclear Science Symposium and Medical Imaging Conference, pp. 867-871, (Edited by L. Klaisner), San Francisco, CA, 1993.

[8] W. W. Moses, J. W. Young, K. Baker, et al., "The electronics system for the LBNL positron emission mammography (PEM) camera," IEEE Trans. Nucl. Sci., vol. NS-48, pp. 632-636, 2001.

[9] C.L. Melcher and J.S. Schweitzer, "Cerium-doped lutetium oxyorthosilicate: a fast, efficient new scintillator," IEEE Trans. Nucl. Sci., vol 39, pp. 502-505, 1992.

[10] Y. Shao, et al., " Dual APD array readout of LSO crystals: Optimization of crystal surface treatment," IEEE Trans. Nucl. Sci., vol. 49, pp. 649-654, 2000 .

[11] E. Gramsch, R. Avila, and P. Bui, "Measurement of the depth of interaction of an LSO scintillator using a planar process APD," IEEE Trans. Nucl. Sci., vol. 50, pp. 307-312, 2003. 